\title{
Maximising Information from Aberration-Corrected STEM images: Applications to Plasmonic, Semiconductor and Battery Materials.
}

\author{
Y Zhu $^{1,2}$, H Katz-Boon ${ }^{2}$, CL Zheng ${ }^{1}$, M Walsh ${ }^{2}$, C Dwyer ${ }^{3}$, L Bourgeois ${ }^{1}$, R Withers ${ }^{4}$ and J Etheridge ${ }^{1,2}$ \\ 1. Monash Centre for Electron Microscopy, Monash University, Victoria 3800, Australia \\ 2. Department of Materials Engineering, Monash University, Victoria 3800, Australia \\ 3. Ernst Ruska-Centre, Peter Gruenberg Inst, Forschungszentrum Juelich, D-52425 Juelich, Germany \\ 4. Research School of Chemistry, Australian National University, ACT 0200, Australia
}

Aberration correction has enabled STEM images to be taken with unprecedented spatial resolution. However, to realise the full potential of these images and maximize the information we can extract about the specimen, we need to measure and quantify the experimental parameters (such as spatial and temporal coherence functions, detector response etc) and understand the electron scattering processes [13]. Armed with this information, we can then tailor the electron-optical conditions to optimize the sensitivity of the image to desired structural information, so that it can be extracted from the image in an efficient, quantitative and practical way. We illustrate this approach with applications to functional materials being developed for plasmonic, opto-electronic and battery applications, for example:

The measurement of the relative stability of different nanofacets on gold nanorods versus their atomic structure and size (Fig 1). Understanding facet stability is vital for understanding growth mechanisms and catalytic behavior, however, there is no established method for measuring the stability of facets that are just a few atoms wide. Using a quantitative analysis of ADF-STEM images to count atoms at successive time intervals and in two different crystal orientations, the crystal facet orientation and stability can be determined. This approach reveals the co-existence of high and low index facets on the same nanorod and, furthermore, that these facets have comparable stability [4].

The measurement of oxygen octahedral tilt angle, unit cell by unit cell, together with the corresponding strain, in the promising $\mathrm{Li}$-ion conducting perovskite $\mathrm{Li}_{\mathbf{x}} \mathrm{Nd}_{1-\mathrm{x}} \mathrm{TiO}_{3}(\mathrm{Fig} 2)$. The nanodomain structure of this material has been the subject of intense debate: is it due to a compositional phase separation or an octahedral tilt modulation [5]? By tuning the detector geometry to optimize the sensitivity of the image to oxygen and Ti positions, the oxygen octahedra can be imaged and the tilt angle measured quantitatively, even in thick crystals $<150 \mathrm{~nm}$ [6]. This reveals directly the modulation of the octahedral tilt angle in a nanodomain formation and enables the measurement of the higher order harmonics associated with this modulation that are not otherwise accessible from diffraction methods.

The measurement of Al composition in beam sensitive AlGaAs/GaAs quantum tube nanowires (Fig 3). By comparing the absolute intensity distribution in experimental ADF-STEM images with a look-up table of intensities versus $\mathrm{Al}$ content calculated using all the measured experimental parameters [1-3], a spatial map of composition can be determined [7,8]. This is a rapid, low dose method, compared with EDS and EELS, ensuring precise, accurate and high resolution measurements.

\section{References:}

[1] C Maunders, C Dwyer, P Tiemeijer, J Etheridge, Ultramicroscopy 111 (2011) 1437

[2] C Dwyer, C Maunders, CL Zheng, M Weyland, P Tiemeijer, J. Etheridge App Phys Lett 100 (2012) 
[3] CL Zheng, J Etheridge Ultramicroscopy 125 (2013) 49

[4] H Katz-Boon et al. Nano Letts just accepted (2015) DOI: 10.1021/acs.nanolett.5b00124

[5] R Erni, et al. Nat. Mater. 13 (2014) 216 ; B Guiton \& P Davies Nat. Mater. 13 (2014) 217

[6] Y Zhu, R Withers, L Bourgeois, C Dwyer, J Etheridge, submitted (2015).

[7] H Kauko, CL Zheng, Y Zhu et al. App Phys Letts 103 (2013) 232111

[8] N Jiang, Q Gao, P Parkinson, J Wong-Leung et al. Nano Lett 13 (2013) 5135

[9] Funding is acknowledged from the Australian Research Council Grants DP110104734,

DP120101573, LE0454166. We thank A. Funston, P. Mulvaney and C. Jagadish for specimens.
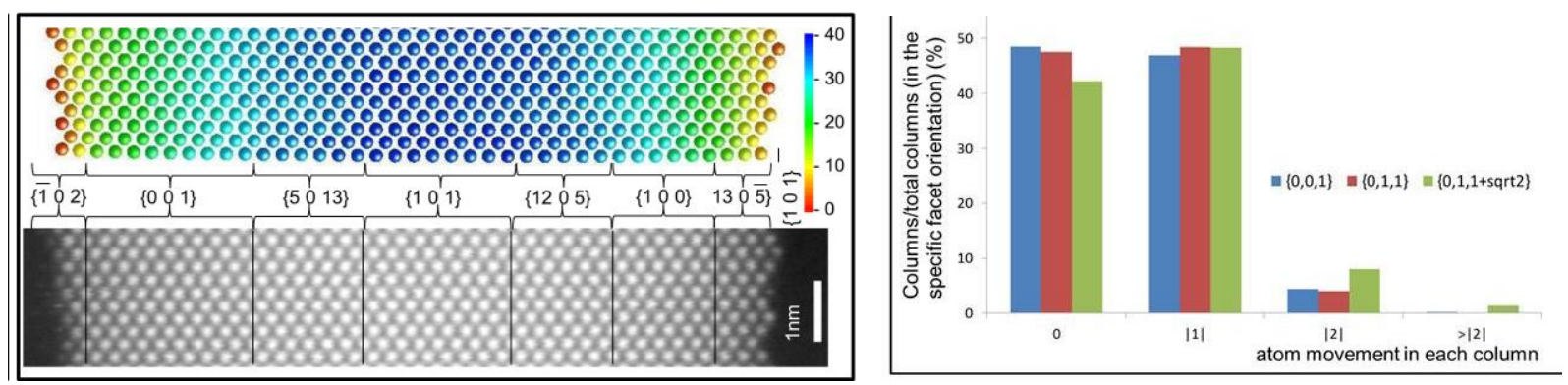

Figure 1. (Left) An ADF-STEM image of a Au nanorod crossection and corresponding "atom map" (top left), part of a time series used to determine the relative stability of crystal nanofacet orientations (right).
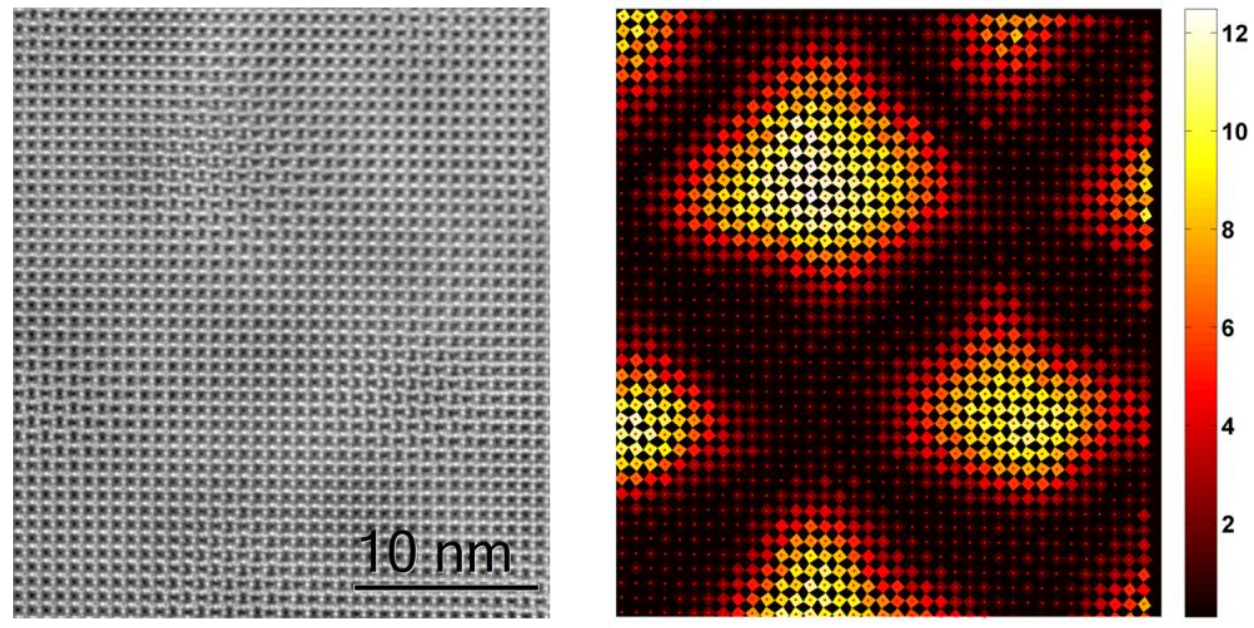

Figure 2. (Right) Map of absolute [001]-tilt angle, unit cell by unit cell, measured from a STEM image (left) with detector geometry optimized to reveal $\mathrm{O}$ and Ti sublattices in $\mathrm{Li}_{0.38} \mathrm{Nd}_{0.54} \mathrm{TiO}_{3}$.
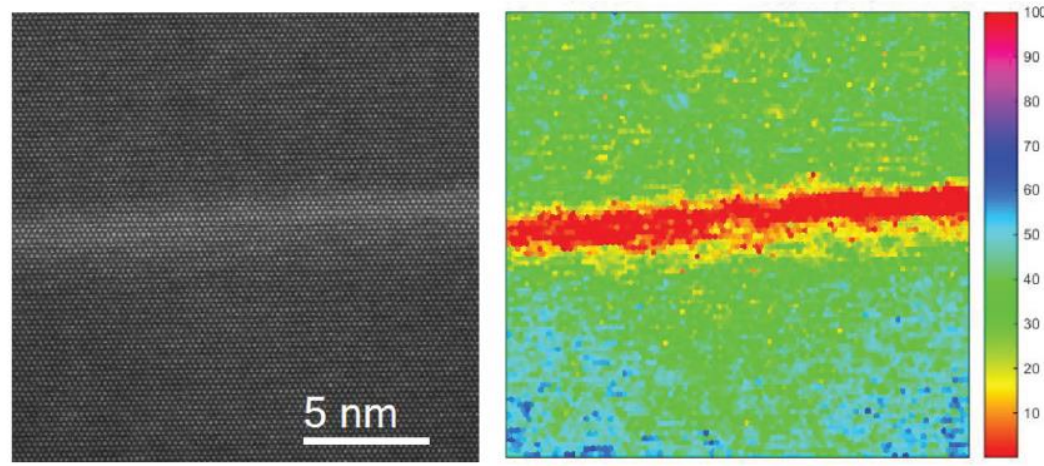

Figure 3. Cross-section of GaAs/AlGaAs quantum well tube heterostructured nanowire. (Left) HAADF image and (right) corresponding $\mathrm{Al}$ composition map. 